\title{
Left Ventricular Diastolic Dysfunction in Ischemic Stroke: Functional and Vascular Outcomes
}

\author{
Hong-Kyun Park, ${ }^{\mathrm{a*}}$ Beom Joon Kim, ${ }^{\mathrm{a} *}$ Chang-Hwan Yoon, ${ }^{\mathrm{b}}$ Mi Hwa Yang, ${ }^{\mathrm{a}}$ Moon-Ku Han, ${ }^{\mathrm{a}}$ \\ Hee-Joon Bae ${ }^{\mathrm{a}}$ \\ ${ }^{a}$ Department of Neurology and Cerebrovascular Center, Seoul National University Bundang Hospital, Seongnam, Korea \\ 'Division of Cardiology, Department of Internal Medicine, Seoul National University Bundang Hospital, Seongnam, Korea
}

\begin{abstract}
Background and Purpose Left ventricular (LV) diastolic dysfunction, developed in relation to myocardial dysfunction and remodeling, is documented in $15 \%-25 \%$ of the population. However, its role in functional recovery and recurrent vascular events after acute ischemic stroke has not been thoroughly investigated.

Methods In this retrospective observational study, we identified 2,827 ischemic stroke cases with adequate echocardiographic evaluations to assess LV diastolic dysfunction within 1 month after the index stroke. The peak transmitral filling velocity/mean mitral annular velocity during early diastole (E/ $\mathrm{e}^{\prime}$ ) was used to estimate LV diastolic dysfunction. We divided patients into 3 groups according to E/e' as follows: $<8,8-15$, and $\geq 15$. Recurrent vascular events and functional recovery were prospectively collected at 3 months and 1 year.

Results Among included patients, E/e' was 10.6 \pm 6.4 : E/e' <8 in 993 (35\%), 8-15 in 1,444 (51\%), and $\geq 15$ in $378(13 \%)$ cases. Functional dependency or death (modified Rankin Scale score $\geq 2$ ) and composite vascular events were documented in 1,298 (46\%) and $187(7 \%)$ patients, respectively, at 3 months. In multivariable analyses, ischemic stroke cases with $\mathrm{E} / \mathrm{e}^{\prime} \geq 15$ had increased odds of functional dependence or death at 3 months (adjusted OR [95\% Cl]: 1.73 [1.27-2.35]) or 1 year (1.47 [1.062.06]) and vascular events within 1 year (1.65 [1.08-2.51]). Subgroups with normal ejection fraction or sinus rhythm exhibited a similar overall pattern and direction.

Conclusions LV diastolic dysfunction was associated with poor functional outcomes and composite vascular events up to 1 year.
\end{abstract}

Keywords Left ventricular diastolic dysfunction; Recurrent vascular event; Functional outcome Acute ischemic stroke

\section{Introduction}

A dysfunction in the diastolic relaxation of the cardiac left ventricle (LV), even in cases without typical symptoms of heart failure, is documented in 15\% to $25 \%$ of the population, and its prevalence increases with age. ${ }^{1-3}$ Currently, LV diastolic dysfunction appears to be a result of myocardial dysfunction and remodeling driven by endothelial inflammation and oxidative stress. ${ }^{4}$

\author{
Correspondence: Moon-Ku Han \\ Department of Neurology and \\ Cerebrovascular Center, Seoul National \\ University Bundang Hospital, \\ 82 Gumi-ro 173beon-gil, Bundang-gu, \\ Seongnam 13620, Korea \\ Tel: +82-31-787-7464 \\ Fax: +82-31-787-4059 \\ E-mail:mkhan@snu.ac.kr \\ Received: December 1, 2015 \\ Revised: March 14, 2016 \\ Accepted: March 21, 2016 \\ *These authors contributed equally to this \\ work. \\ This work was supported by Grant No. \\ 02-2014-039 of the SNUBH Research \\ Fund and a grant from the Korea \\ Healthcare Technology R\&D Project, \\ Ministry of Health and Welfare, Republic \\ of Korea (HI10C2020). \\ The authors have no financial conflicts of \\ interest.
}

The importance of LV diastolic dysfunction is clear because even after controlling for comorbidities, it has been associated with the development of heart failure, future cardiovascular events, and increased mortality, even in asymptomatic subjects. ${ }^{5-7}$

For stroke physicians, LV diastolic dysfunction has important clinical implications. A major symptom of LV diastolic dysfunction is exercise intolerance; ${ }_{i}^{8}$ however, stroke survivors might not have enough exercise capacity when they have LV diastolic dys- 
function. Therefore, their exercise prescriptions must be modified. Additionally, the individual risk for recurrent stroke or vascular events may be altered by the presence of asymptomatic LV diastolic dysfunction. However, its clinical implication has only been studied in relation to a potential source of cryptogenic stroke, ${ }^{9}$ although LV diastolic dysfunction could be evaluated by a routine cardiac echocardiography, which is widely available. ${ }^{10,11}$

In this context, we hypothesized that LV diastolic dysfunction would have deleterious effects on functional recovery and vascular events after ischemic stroke. Thus, we attempted to address this question by analyzing approximately 3,000 consecutive ischemic stroke cases with prospectively collected outcome information.

\section{Methods}

Of 4,808 stroke patients admitted to the Seoul National University Bundang Hospital within 7 days after symptom onset between August 2007 and June 2013, we selected ischemic stroke cases whose ischemic lesions were documented by relevant neuroimaging studies $(N=3,858)$ and subjects who underwent echocardiographic evaluation within 1 month from the index stroke $(N=3,030)$. We excluded cases with inadequate echocardiographic data to evaluate the LV diastolic function $(N=203)$. The analysis dataset consisted of 2,827 acute ischemic stroke patients with echocardiography data. This is a retrospective study undertaken for the quality improvement of stroke management, using a prospectively collected cohort. All participants in this study gave their informed consent. In addition, the study protocol conforms to the ethical guidelines of the 1975 Declaration of Helsinki as reflected in a priori approval by the institution's human research committee. Finally, this study was approved by the Institutional Review Board at the Seoul National University Bundang Hospital [IRB approval No. B-1501-284-102].

We collected baseline demographic data and clinical information for all study participants, including age, sex, premorbid functional status, history of previous stroke, mechanism of ischemic stroke, National Institute of Health Stroke Scale (NIHSS) score, acute reperfusion treatment, medication at discharge, and cardiovascular risk factors. Cardiovascular risk factors included hypertension (previous use of antihypertensive medication, systolic blood pressure $>140 \mathrm{mmHg}$, or diastolic pressure $>90$ $\mathrm{mmHg}$ at discharge); diabetes mellitus (previous use of glucoselowering medication or hemoglobin A1c $\geq 6.5 \%$ ); dyslipidemia (previous use of lipid-lowering medication, fasting low density lipoprotein cholesterol $>160 \mathrm{mg} / \mathrm{dL}$, or fasting total cholesterol $>240 \mathrm{mg} / \mathrm{dL}$ ); habitual smoking (current or past regular smoking); and atrial fibrillation. We also obtained laboratory informa- tion, including random glucose at presentation, hemoglobin, total cholesterol, and initial systolic and diastolic blood pressures.

A well-trained and experienced nurse (M-H. Y) prospectively collected the functional outcome status (modified Rankin Scale [mRS] scores) and vascular events after the index stroke through telephone interviews with the patients or their immediate caregivers or at their routine clinic visit. Prospective outcomes were determined at 3 months and 1 year after the index stroke. The missing prospective outcomes were as follows: $15 \mathrm{mRS}$ scores and 0 vascular events at 3 months, and $316 \mathrm{mRS}$ scores and 275 vascular events at 1 year.

In our institution, acute ischemic stroke patients undergo an echocardiographic evaluation to identify potential embolic sources and the coexistence of ischemic heart disease as part of the clinical routine, if the patients or their caregivers do not refuse the examination due to any reasons such as personal economic issues and poor cooperation. Echocardiographic data were extracted in a retrospective manner through the review of medical records. Experienced technicians and board-certified cardiologists, who were not aware of the study purposes or the patient outcomes, performed and interpreted transthoracic echocardiographic evaluations in accordance with the published and relevant current guidelines. ${ }^{12}$ In brief, LV dimensions, both at end-diastole and at systole, were measured at the standard parasternal short-axis view of the papillary muscle level or parasternal longaxis view. The dimensions of the aortic root, sinotubular junction, and ascending thoracic aorta diameter were measured at the standard parasternal long-axis view. Peak early transmitral filling velocities during the early diastolic $(E)$, late diastole $(A)$ and deceleration time (DT) were imaged in the apical 4-chamber view at the tip of the mitral leaflets. A Tissue Doppler image was applied to the apical 4-chamber view to determine the mean early $\left(e^{\prime}\right)$ and late (a') velocities at septal mitral annuli. The maximal left atrial (LA) volume was indexed to the body surface area to measure the $L A$ volume index.

The E/e' ratio has been proposed as a surrogate for LA pressure and plays a prominent role for the assessment of diastolic function in the guidelines. Therefore, we used the E/e' ratio as the primary grading system to estimate the LV diastolic functional status, classifying the E/e' ratio as $<8,8-15$, and $\geq 15 .{ }^{13}$ To validate the results further, we also adopted another grading system and separated the patients into 4 groups in accordance with the current recommendations.13 Normal LV diastolic function was defined by the presence of an e' $\geq 8 \mathrm{~cm} / \mathrm{s}$, E/A ratio $0.8-1.5$, DT $160-200 \mathrm{~ms}$, and LA volume index $<34$. Grade I diastolic dysfunction represented an $\mathrm{e}^{\prime}<8 \mathrm{~cm} / \mathrm{s}$, E/e' $<8$, E/A ratio $<0.8$, and DT $>200 \mathrm{~ms}$. An E/A ratio 0.8-1.5, e' $<8 \mathrm{~cm} / \mathrm{s}$, E/e' 8-15, and DT 160-200 ms were defined as grade II diastolic dysfunc- 
tion (pseudo-normal pattern). The most severe form of LV diastolic dysfunction (grade III) occurs with an E/e' $\geq 15$, E/A ratio $\geq 2$, and DT $<160 \mathrm{~ms}$. To classify the status of LV diastolic function in the cases with an E/A ratio $0.8-1.5$ or DT $160-200$ ms, which might be observed in both normal diastolic function and grade II diastolic dysfunction, other parameters such as e', LA volume index, and E/e' ratio were used to determine the grade of diastolic function. Because the peak $A$ velocity is unmeasurable in atrial fibrillation patients, we did not apply the E/A ratio for the grading of LV diastolic dysfunction in these cases. However, $e^{\prime}$, DT, and E/e', which are not significantly influenced by atrial performance, were used as parameters to measure LV diastolic function. Normal LV diastolic function was defined by the presence of an $\mathrm{e}^{\prime} \geq 8 \mathrm{~cm} / \mathrm{s}$ and DT 160-200 ms. An E/e' $<8, \mathrm{e}^{\prime}<8$ $\mathrm{cm} / \mathrm{s}$, and DT $>200 \mathrm{~ms}$ were defined as grade I diastolic dysfunction. For grade II dysfunction, the criteria were DT 160-200 $\mathrm{ms}, \mathrm{e}^{\prime}<8 \mathrm{~cm} / \mathrm{s}$, and E/e' $8-15$. The patients with grade III dysfunction had a DT $<160 \mathrm{~ms}, \mathrm{e}^{\prime}<8 \mathrm{~cm} / \mathrm{s}$, and E/e' $\geq 15$.

The categorical variables are presented as frequencies (percentages), and the continuous variables are presented as the means \pm standard deviations or medians [interquartile ranges], as appropriate. The baseline characteristics were compared using chi-squared tests for the categorical variables and $t$ tests for the continuous variables. In the current analyses, functional dependency or death (a mRS score of 2-6), recurrent strokes, and composite vascular events, including recurrent stroke, myocardial infarction, and vascular death (any death directly attributed from vascular events), were designated as major outcome variables at 3 months and 1 year after the index stroke. We constructed multivariable binary logistic regression models that included the major outcome variables as dependent variables and adjusted for age, sex, recanalization treatment, initial stroke severity, mechanism of ischemic stroke, hypertension, diabetes, dyslipidemia, habitual smoking, atrial fibrillation, hemoglobin, glucose level at presentation, total cholesterol, and pre-morbid mRS score. We also analyzed the pre-specified ordinal logistic regression models that included the continuous scale of the mRS scores as dependent variables. Thus, this analysis strategy appears to be more sensitive to the minor influence of predictor variables than classical binary logistics. ${ }^{14}$ Additionally, we performed additional multivariable binary logistic regression analyses in several subgroups as follows: those with preserved ejection fraction, those without atrial fibrillation, and those who underwent echocardiographic evaluation after symptom onset. The balance of the baseline characteristics among the groups might not be obtained, and age and initial stroke severity might influence functional outcomes. Therefore, we divided the total cases into 4 groups using the median values of age and initial NIHSS score.
We performed a stratified analysis on the following subgroups: 1) age $<69$ and NIHSS score $<3 ; 2$ ) age $\geq 69$ and NIHSS score $<3$; 3) age $<69$ and NIHSS score $\geq 3$; and 4 ) age $\geq 69$ and NlHSS score $\geq 3$. Each outcome index was analyzed using the available cases. We excluded from the analyses the cases whose clinical outcome information was unobtainable. Significance levels were set at a 2-tailed $P$ value of $<0.05$. Statistical analyses were performed using Stata 13 (StataCorp LP, College Station, TX, USA).

\section{Results}

Of the 2,827 included subjects, 1,724 (61\%) cases were male, and the mean age was $67 \pm 13$ years old. The NIHSS score at presentation was 3 [1-6], and recanalization treatment was performed in $383(14 \%)$ cases. The prevalence of major vascular risk factors was 69\% hypertension, 31\% diabetes, 24\% dyslipidemia, $44 \%$ habitual smoking, and 15\% atrial fibrillation. Among the included subjects, 2,651 (94\%) cases had echocardiography performed within 7 days from stroke onset. The mean time from stroke onset to echocardiography was $1.99 \pm 3.54$ days. The mean E/e' across all cases was $10.6 \pm 6.4$; with an E/e' $<8$ occurring in $993(35 \%)$ cases, 8-15 occurring in 1,444 (51\%) cases, and $\geq 15$ occurring in $378(13 \%)$ cases. The echocardiographic grading of the LV diastolic dysfunction classified the cases into grade I in 41\% $(1,161)$, grade II in 12\% (332), and grade III in 6\% (181) of the included population. Echocardiographic profiles of the included subjects are as follows: $61.8 \pm 7.7 \%$ for ejection fraction, $66.64 \pm 25.52 \mathrm{~cm} / \mathrm{s}$ for $E, 78.38 \pm 20.62 \mathrm{~cm} / \mathrm{s}$ for $A$, $5.31 \pm 3.44 \mathrm{~cm} / \mathrm{s}$ for e', $9.48 \pm 2.38 \mathrm{~cm} / \mathrm{s}$ for a', $206.61 \pm 71.68 \mathrm{~ms}$ for DT, $38.80 \pm 19.97 \mathrm{~mL} / \mathrm{m}^{2}$ for LA volume index, and $1.31 \pm 0.40$ for mean pulmonary artery systolic pressure; the end systolic and diastolic dimensions of LV were $29.54 \pm 5.86$ and $46.56 \pm 5.65$ $\mathrm{mm}$, respectively. A regional wall motion abnormality was observed in $225(8 \%)$ cases. Among the 936 (33\%) cases who underwent the transesophageal echocardiographic evaluation, there were 13 patients with thrombi and 44 patients with spontaneous echo contrast in their LA cavity or appendage (Supplemental Table 1). Functional dependency or death was documented in 1,298 (46\%) cases at 3 months and 1,017 (41\%) cases at 1 year. Recurrent stroke occurred in $171(6 \%)$ cases at 3 months and $215(8 \%)$ cases at 1 year. The composite vascular events, including recurrent stroke, myocardial infarction, and vascular death, were documented in 187 (7\%) cases at 3 months and $250(10 \%)$ cases at 1 year. Compared with the patients with an E/e' $<8$, the patients with an E/e' $\geq 15$ were more likely to be female, old, and exhibit more prevalent vascular risk factors and prominent neurological deficits (Table 1). The prevalence of func- 
Table 1. Baseline characteristics of study population via the E/e' level

\begin{tabular}{|c|c|c|c|c|}
\hline \multirow{2}{*}{ Variables } & \multicolumn{3}{|c|}{ Level of E/e' } & \multirow{2}{*}{$P$ for difference } \\
\hline & $<8[N=993,35 \%]$ & $8-15[N=1444,51 \%]$ & $\geq 15[\mathrm{~N}=378,13 \%]$ & \\
\hline \multicolumn{5}{|l|}{ Demographic information } \\
\hline Male & $685(69 \%)$ & $875(61 \%)$ & $159(42 \%)$ & $<0.01$ \\
\hline Age & $64 \pm 14$ & $67 \pm 12$ & $73 \pm 12$ & $<0.01$ \\
\hline \multicolumn{5}{|l|}{ Vascular risk factors } \\
\hline Stroke & $166(17 \%)$ & $257(18 \%)$ & $73(19 \%)$ & 0.51 \\
\hline Hypertension & $612(62 \%)$ & $1,043(72 \%)$ & 300 (79\%) & $<0.01$ \\
\hline Diabetes & $255(26 \%)$ & $472(33 \%)$ & $154(41 \%)$ & $<0.01$ \\
\hline Hyperlipidemia & $202(20 \%)$ & $372(26 \%)$ & $102(27 \%)$ & $<0.01$ \\
\hline Atrial fibrillation & $88(9 \%)$ & $229(16 \%)$ & $114(30 \%)$ & $<0.01$ \\
\hline Habitual smoking & $483(49 \%)$ & $634(44 \%)$ & $116(31 \%)$ & $<0.01$ \\
\hline Coronary heart disease & $56(7 \%)$ & $110(8 \%)$ & $60(16 \%)$ & $<0.01$ \\
\hline Congestive heart failure & $1(0.1 \%)$ & $6(0.4 \%)$ & $6(1.6 \%)$ & $<0.01$ \\
\hline Mechanism of ischemic stroke & & & & $<0.01$ \\
\hline Large artery atherosclerosis & $427(43 \%)$ & $578(40 \%)$ & $114(30 \%)$ & \\
\hline Small vessel occlusion & $198(20 \%)$ & $273(19 \%)$ & $55(15 \%)$ & \\
\hline Cardioembolism & $150(15 \%)$ & $313(22 \%)$ & $145(38 \%)$ & \\
\hline Other determined etiology & $43(4 \%)$ & $43(3 \%)$ & $5(1 \%)$ & \\
\hline Undetermined etiology & $175(18 \%)$ & $237(16 \%)$ & $59(16 \%)$ & \\
\hline \multicolumn{5}{|l|}{ Stroke information } \\
\hline $\mathrm{mRS}$ score 0 before index stroke & $871(88 \%)$ & $1,259(87 \%)$ & 319 (84\%) & 0.25 \\
\hline NIHSS at initial presentation & $3[1-6]$ & $3[1-6]$ & $4[2-9]$ & $<0.01$ \\
\hline Initial systolic blood pressure $(\mathrm{mmHg})$ & $154 \pm 26$ & $155 \pm 27$ & $155 \pm 28$ & 0.92 \\
\hline Initial random glucose (mg/dL) & $141 \pm 67$ & $144 \pm 64$ & $151 \pm 68$ & 0.02 \\
\hline Hemoglobin $(\mathrm{g} / \mathrm{dL})$ & $14 \pm 2$ & $14 \pm 2$ & $13 \pm 2$ & $<0.01$ \\
\hline $\mathrm{HbA1c}(\%)$ & $6.3 \pm 1.3$ & $6.4 \pm 1.3$ & $6.5 \pm 1.4$ & $<0.01$ \\
\hline Total cholesterol (mg/dL) & $184 \pm 44$ & $179 \pm 42$ & $173 \pm 41$ & $<0.01$ \\
\hline Hyperacute recanalization treatment & $121(12 \%)$ & $188(13 \%)$ & $72(19 \%)$ & $<0.01$ \\
\hline \multicolumn{5}{|l|}{ Echocardiographic profile } \\
\hline Ejection fraction (\%) & $62.6 \pm 6.5$ & $62.2 \pm 7.2$ & $59.0 \pm 10.7$ & $<0.01$ \\
\hline Preserved ejection fraction ( $\geq 40 \%$ ) & 977 (99\%) & $1,416(98 \%)$ & $350(94 \%)$ & $<0.01$ \\
\hline $\mathrm{E}(\mathrm{cm} / \mathrm{s})$ & $56.6 \pm 15.2$ & $67.6 \pm 18.5$ & $89.4 \pm 46.4$ & $<0.01$ \\
\hline$A(\mathrm{~cm} / \mathrm{s})$ & $72.4 \pm 17.5$ & $80.1 \pm 18.2$ & $89.5 \pm 3.11$ & $<0.01$ \\
\hline $\mathrm{e}^{\prime}(\mathrm{cm} / \mathrm{s})$ & $5.3 \pm 4.7$ & $5.6 \pm 2.5$ & $4.1 \pm 1.7$ & $<0.01$ \\
\hline$a^{\prime}(\mathrm{cm} / \mathrm{s})$ & $10.8 \pm 2.6$ & $9.3 \pm 2.0$ & $7.7 \pm 2.0$ & $<0.01$ \\
\hline Deceleration time (msec) & $203.9 \pm 61.3$ & $203.7 \pm 70.4$ & $225.2 \pm 95.5$ & $<0.01$ \\
\hline Left atrial volume index $\left(\mathrm{mm} / \mathrm{m}^{2}\right)$ & $32.2 \pm 11.9$ & $37.4 \pm 17.2$ & $52.3 \pm 29.0$ & $<0.01$ \\
\hline Left ventricular end systolic dimension (mm) & $29.2 \pm 5.5$ & $29.9 \pm 6.1$ & $29.0 \pm 5.8$ & $<0.01$ \\
\hline Left ventricular end diastolic dimension $(\mathrm{mm})$ & $46.6 \pm 5.5$ & $46.7 \pm 5.7$ & $46.0 \pm 5.8$ & 0.11 \\
\hline Mitral stenosis & $0(0 \%)$ & $2(0 \%)$ & $16(4 \%)$ & $<0.01$ \\
\hline Mitral regurgitation & $455(46 \%)$ & 759 (53\%) & $242(64 \%)$ & $<0.01$ \\
\hline Aortic stenosis & $11(1 \%)$ & $24(2 \%)$ & $15(4 \%)$ & $<0.01$ \\
\hline Aortic regurgitation & $255(26 \%)$ & $442(31 \%)$ & $172(46 \%)$ & $<0.01$ \\
\hline Hypertrophic cardiomyopathy & $6(1 \%)$ & $15(1 \%)$ & $14(4 \%)$ & $<0.01$ \\
\hline \multicolumn{5}{|l|}{ Discharge medication } \\
\hline Anti-diabetic agents & $161(20 \%)$ & $346(25 \%)$ & $112(31 \%)$ & $<0.01$ \\
\hline Anti-hypertensive agents & $338(41 \%)$ & 749 (54\%) & $219(60 \%)$ & $<0.01$ \\
\hline Anti-hyperlipidemic agents & 608 (74\%) & $1,180(85 \%)$ & 304 (84\%) & $<0.01$ \\
\hline Anti-platelet agents & 851 (87\%) & 1,180 (83\%) & $276(75 \%)$ & $<0.01$ \\
\hline Anticoagulant & $122(12 \%)$ & $283(20 \%)$ & $107(29 \%)$ & $<0.01$ \\
\hline
\end{tabular}

mRS, modified Rankin Scale; NIHSS, National Institute of Health Stroke Scale; composite vascular events include recurrent stroke, myocardial infarction and vascular death after the index stroke; $E$, peak early transmitral filling velocities during early diastole; $A$, peak early transmitral filling velocities during late diastole; e', mean early velocities at septal mitral annuli on Tissue Doppler image; a', mean late velocities at septal mitral annuli on Tissue Doppler image. 
Table 2. Clinical outcomes according to the E/e' level

\begin{tabular}{|c|c|c|c|c|}
\hline \multirow{2}{*}{ Variables } & \multicolumn{3}{|c|}{ Level of E/e' } & \multirow{2}{*}{$P$ for difference } \\
\hline & $<8[N=993,35 \%]$ & $8-15[N=1444,51 \%]$ & $\geq 15[\mathrm{~N}=378,13 \%]$ & \\
\hline \multicolumn{5}{|c|}{ Outcomes at 3 months after stroke* } \\
\hline $\mathrm{mRS}$ score 2-6 & $433(43 \%)$ & $677(45 \%)$ & $254(63 \%)$ & $<0.01$ \\
\hline Recurrent stroke & $51(5 \%)$ & $99(7 \%)$ & $30(7 \%)$ & 0.13 \\
\hline Composite vascular events & $60(6 \%)$ & $104(7 \%)$ & $34(8 \%)$ & 0.21 \\
\hline \multicolumn{5}{|l|}{ Outcomes at 1 year after stroke ${ }^{\dagger}$} \\
\hline mRS score 2-6 & $353(38 \%)$ & $521(40 \%)$ & 201 (58\%) & $<0.01$ \\
\hline Recurrent stroke & $67(7 \%)$ & $116(9 \%)$ & $42(12 \%)$ & 0.03 \\
\hline Composite vascular events & $79(8 \%)$ & $129(10 \%)$ & $56(16 \%)$ & $<0.01$ \\
\hline
\end{tabular}

mRS, modified Rankin Scale; composite vascular events include recurrent stroke, myocardial infarction and vascular death after the index stroke; $E_{1}$ peak early transmitral filling velocities during early diastole; $e^{\prime}$, mean early velocities at septal mitral annuli on Tissue Doppler image.

${ }^{*}$ Outcomes at 3 months after stroke were based on 2,812 cases for the mRS score and 2,827 cases for recurrent stroke and composite vascular events; ${ }^{\dagger}$ Outcomes at 1 year after stroke were based on 2,511 cases for the mRS score and 2,552 cases for recurrent stroke and composite vascular events.

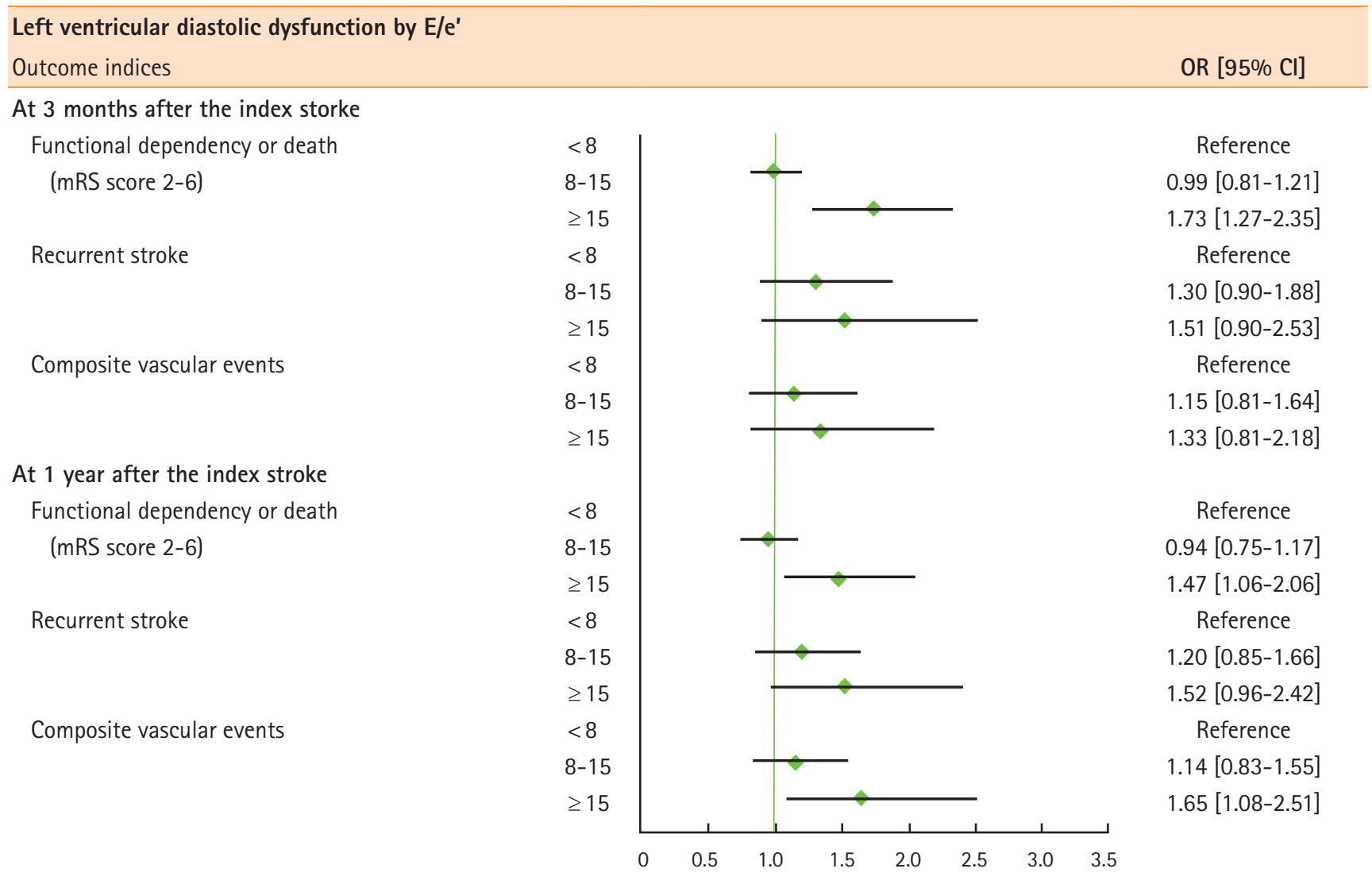

Figure 1. Multivariable logistic regression analyses between left ventricular diastolic dysfunction (E/e') and functional recovery and event outcomes. Composite vascular events include recurrent stroke, myocardial infarction, and vascular death after the index stroke.

$\mathrm{OR}$, odds ratio; $\mathrm{Cl}$, confidence interval; $\mathrm{mRS}$, modified Rankin Scale.

tional dependency or death showed significant differences according to the level of E/e' at both 3 months and 1 year. However, there were statistical differences in the event outcomes only at 1 year after the index stroke (Table 2). Echocardiographic profiles also differed according to the E/e' level. Ischemic stroke patients with a higher E/e' ratio also exhibited prevalent vascular risk factors (Supplemental Table 2). The prevalence of clinical outcomes is demonstrated in Supplemental Table 3. The distribution of possible cardioembolic sources revealed by the transesophageal echocardiographic evaluation is summarized in Supplemental Table 1.

The ischemic stroke cases with a baseline E/e' $\geq 15$ were significantly associated with functional dependence or death (mRS score $\geq 2$ ) at 3 months (adjusted OR [95\% Cl]: 1.73 [1.27-2.35]) 
Table 3. Sensitivity analyses via the E/e' level

\begin{tabular}{|c|c|c|}
\hline \multirow{2}{*}{ Analytic strategies } & \multicolumn{2}{|c|}{ Marker of diastolic dysfunction } \\
\hline & E/e' & OR $[95 \% \mathrm{Cl}]$ \\
\hline \multicolumn{3}{|c|}{$\begin{array}{l}\text { Ordinal logistic regression models (continuous scale of mRS score as } \\
\text { dependent variable) }\end{array}$} \\
\hline \multirow[t]{3}{*}{$\mathrm{mRS}$ score at 3 months } & $<8$ & Reference \\
\hline & 8-15 & $1.00[0.86-1.17]$ \\
\hline & $\geq 15$ & $1.58[1.24-2.00]$ \\
\hline \multirow[t]{3}{*}{$\mathrm{mRS}$ score at 1 year } & $<8$ & Reference \\
\hline & $8-15$ & 1.00 [0.84-1.18] \\
\hline & $\geq 15$ & $1.58[1.22-2.04]$ \\
\hline \multicolumn{3}{|c|}{ Subjects with preserved ejection fraction $(N=2,751)$} \\
\hline \multirow[t]{2}{*}{$\begin{array}{l}\text { Functional dependency or death } \\
\text { at } 3 \text { months (mRS score 2-6) }\end{array}$} & $\begin{array}{l}<8 \\
8-15\end{array}$ & $\begin{array}{c}\text { Reference } \\
0.98[0.80-1.20]\end{array}$ \\
\hline & $\geq 15$ & $1.69[1.23-2.32]$ \\
\hline \multirow[t]{2}{*}{$\begin{array}{l}\text { Composite vascular events at } \\
3 \text { months }\end{array}$} & $\begin{array}{l}<8 \\
8-15\end{array}$ & $\begin{array}{c}\text { Reference } \\
1.16[0.82-1.65]\end{array}$ \\
\hline & $\geq 15$ & $1.33[0.80-2.21]$ \\
\hline \multirow[t]{2}{*}{$\begin{array}{l}\text { Functional dependency or death } \\
\text { at } 1 \text { year (mRS score 2-6) }\end{array}$} & $\begin{array}{l}<8 \\
8-15\end{array}$ & $\begin{array}{c}\text { Reference } \\
0.94[0.75-1.17]\end{array}$ \\
\hline & $\geq 15$ & $1.39[0.99-1.97]$ \\
\hline \multirow{3}{*}{$\begin{array}{l}\text { Composite vascular events at } \\
1 \text { year }\end{array}$} & $<8$ & Reference \\
\hline & $8-15$ & $1.16[0.84-1.59]$ \\
\hline & $\geq 15$ & $1.68[1.08-2.60]$ \\
\hline \multicolumn{3}{|c|}{ Subjects without atrial fibrillation $(\mathrm{N}=2,394)$} \\
\hline \multirow[t]{2}{*}{$\begin{array}{l}\text { Functional dependency or death } \\
\text { at } 3 \text { months (mRS score 2-6) }\end{array}$} & $\begin{array}{l}<8 \\
8-15\end{array}$ & $\begin{array}{c}\text { Reference } \\
0.94[0.76-1.17]\end{array}$ \\
\hline & $\geq 15$ & $1.77[1.26-2.50]$ \\
\hline \multirow[t]{2}{*}{$\begin{array}{l}\text { Composite vascular events at } \\
3 \text { months }\end{array}$} & $\begin{array}{l}<8 \\
8-15\end{array}$ & $\begin{array}{c}\text { Reference } \\
1.16[0.80-1.69]\end{array}$ \\
\hline & $\geq 15$ & 1.36 [0.78-2.39] \\
\hline \multirow{3}{*}{$\begin{array}{l}\text { Functional dependency or death } \\
\text { at } 1 \text { year (mRS score 2-6) }\end{array}$} & $<8$ & Reference \\
\hline & $8-15$ & $0.89[0.70-1.12]$ \\
\hline & $\geq 15$ & $1.37[0.95-1.98]$ \\
\hline \multirow{3}{*}{$\begin{array}{l}\text { Composite vascular events at } \\
1 \text { year }\end{array}$} & $<8$ & Reference \\
\hline & 8-15 & $1.10[0.79-1.54]$ \\
\hline & $\geq 15$ & $1.88[1.17-3.00]$ \\
\hline
\end{tabular}

Multivariable models were adjusted for age, sex, hyperacute recanalization treatment, mechanism of ischemic stroke, hypertension, diabetes, hyperlipidemia, habitual smoking, atrial fibrillation, hemoglobin, initial random glucose, total cholesterol, NIHSS at initial presentation, and mRS score before the index stroke.

$\mathrm{LV}$, left ventricle; $\mathrm{OR}$, odds ratio; $\mathrm{Cl}$, confidence interval; $\mathrm{mRS}$, modified Rankin Scale; composite vascular events include recurrent stroke, myocardial infarction and vascular death after the index stroke.

and 1 year (1.47 [1.06-2.06]) compared with the cases with an E/e' - $<8$ (Figure 1). An E/e' $\geq 15$ also indicated increased odds of recurrent vascular events until 1 year after stroke (1.65 [1.082.51]); however, the associations with the 3-month event outcomes failed to reach statistical significance. Grade III in echocardiographic grading had similar associations with functional recovery, including poor functional outcomes at 3 months (1.68 [1.13-2.49]) and 1 year (2.01 [1.31-3.07]). However, echocardiographic grading was not associated with event outcomes (Supplemental Table 4).
We performed several pre-specified sensitivity analyses to address the robustness and uncertainty of the main analyses (Table 3 and Supplemental Table 4). The ordinal logistic models consistently indicated that the putative LV diastolic dysfunction was associated with functional dependency after ischemic stroke. To address the precarious methodology in the measurement of diastolic dysfunction, we repeated the main analyses in the subgroups with a preserved ejection fraction or without atrial fibrillation. The overall pattern, direction, and strength of the association between diastolic dysfunction and outcomes after stroke were also comparable in the subsequent analyses.

We further divided our subjects into 4 subgroups by their age $(<69$ and $\geq 69$ years old) and neurological severity (NIHSS score $<3$ and $\geq 3$ ). E/e' 8-15 was not associated with poor clinical outcomes. We found that the overall direction of associations between $\mathrm{E} / \mathrm{e}^{\prime} \geq 15$ and the clinical outcome indices were maintained throughout the subgroups, except for the group with age $\geq 69$ and NIHSS score $\geq 3$ at 3 months, although this finding lost statistical significance due to a decreased number of subjects (Supplemental Table 5).

\section{Discussion}

In our ischemic stroke population, more than half of the cases exhibited LV diastolic dysfunction, and approximately 10\% of the patients exhibited profound dysfunction. LV diastolic dysfunction deteriorated the functional status of the patient over the course of stroke recovery and may have increased the risk of recurrent vascular events in ischemic stroke survivors.

Functional recovery at 3 months or 1 year after stroke was consistently hampered by the presence of LV diastolic dysfunction at baseline, both from the main dichotomous analyses and the additional shift analysis strategies. More than half of stroke survivors achieve functional independence 1 year after the index stroke. ${ }^{10}$ However, stroke survivors face several obvious barriers, including physical disabilities, which function against their expected recovery. ${ }^{15}$ Myocardial remodeling from endothelial inflammation and low nitric oxide bioavailability may cause decreased distensibility in the LV diastole period and interfere with the LV filling process with slow relaxation. ${ }^{16-18}$ In clinical aspects, in addition to low intensity of exercise, early supervised aerobic training and more intensive mobilization also have been exercise goals for functional recovery after stroke. ${ }^{19}$ Thus, LV diastolic dysfunction, even in asymptomatic cases, may impede exercise capacity, ${ }_{1}^{20}$ which is a vital requirement for functional recovery after stroke. ${ }^{21}$

To prove the robustness of the documented association between LV diastolic dysfunction and outcomes after stroke, we 
performed several sensitivity analyses via the incorporation of an additional statistical model and cardiac subgroups. The overall pattern of the associations remained in the sensitivity analyses, such as a poor functional recovery from the advanced stages of LV diastolic dysfunction but increased event outcomes documented only in the extreme group of E/e' values. LV diastolic dysfunction supposedly has a direct impact on recurrent events through impaired left atrial contractility and intracardiac stasis, both of which increase the possibility of thromboembolism. ${ }^{22}$

Several points should be clarified regarding our study. First, among various indicators, we mainly used E/e' to evaluate LV diastolic dysfunction, with the echocardiographic grading system as a supplementary tool, both of which had only marginal correlations (Supplemental Table 6). Because of the complex pathophysiology of diastole, ${ }_{1}^{23}$ a gold standard indicator for diastolic dysfunction has not been devised. A current review article supports uniform integrative multiparametric grading of diastolic function as recommended by the updated guidelines, but establishment of a uniform grading system is considered to be a challenge. ${ }^{13,23,24}$ However, the key indices that are included for grading the diastolic function still yield discrepant information. Among the various echocardiographic markers representing diastolic function, E/e' has been proposed as a surrogate for LA pressure and plays a prominent role in the guidelines. ${ }^{13}$ In addition, E/e' might be a feasible echocardiographic parameter for LV diastolic dysfunction even in the subjects with atrial fibrillation. Since the peak $A$ velocity is unmeasurable in patients with atrial fibrillation, the value of the E/A ratio cannot be applied alone in these cases. ${ }^{13}$ Other parameters are usually considered as supporting parameters of main parameters such as E/e' and E/A. This result implies that a considerable amount of case mix exists in the LV diastolic dysfunction grading system and suggests that the development of more precise tools for investigating LV diastolic dysfunction is necessary. Until any tangible and intuitive parameter for ischemic stroke patients is devised, stroke physicians should be aware of this intricacy and maximally utilize the available echocardiographic parameters. Second, although all the echocardiographic findings were collected from all the patients who had been examined, 113 cases who underwent echocardiographic evaluation before stroke onset were excluded from the study, and resting 715 cases did not have echocardiographic evaluation at baseline. The retrospective design of this study might produce selection bias. Third, we do not have detailed information regarding the pharmacological or rehabilitation interventions after discharge, which may confound the association between LV diastolic dysfunction and stroke outcomes. Fourth, since we included the patients who underwent echocardiographic evaluation after the index stroke, there is the possibility of stroke-induced cardiac dysfunction such as myocytolysis or Takotsubo cardiomyopathy, which might be reversible. In addition, echocardiographic profiles at the acute stage of stroke might be different from late period due to various factors, e.g., fluid therapy, discontinuation of antihypertensive agents, and sympathetic activation. Although early diastolic LV wall or mitral annular velocity and LV filling flow propagation velocity might be relatively volume load-independent, ${ }^{25}$ a combination of other factors possibly influence the cardiac diastolic function. Thus, follow up echocardiography at a later stage would give more information on the association between LV diastolic dysfunction and clinical outcomes. Fifth, since the results of our study were derived from only an Asian population, validations in other ethnic groups might be necessary. Sixth, although initial stroke severity and age were included in multivariable models as covariates, the balance of baseline characteristics among the 3 groups might not be achieved. We performed additional stratified analyses by using the median values of age and initial NIHSS score (Supplemental Table 5). However, the results showed statistical insignificance or a wide range in the confidence interval, which might be influenced by low event rates of each outcome variable or other cardiac function status. Seventh, besides the history of atrial fibrillation that we included as a covariate in multivariable analyses, we need to consider possible effects of other cardiac function variables on the clinical outcomes.

\section{Conclusions}

From our study, we documented that LV diastolic dysfunction was associated with poor functional outcome and composite vascular events up to 1 year (independently of clinical variables including age and baseline NIHSS score). These findings suggest that echocardiographic evaluations for stroke cases, which are typically performed for etiologic work-ups, may provide potentially important prognostic factors for patients and mechanistic insights into functional recovery and recurrence after stroke. In addition, exercise prescriptions and rehabilitation therapy might need modification depending on whether LV diastolic dysfunction is present or not. Further studies in stroke victims that examine recent advances in pharmacological or exercise interventions to prevent functional deterioration or recurrent events are warranted. ${ }^{26,27}$

\section{References}

1. Fischer $M$, Baessler $A$, Hense HW, Hengstenberg $C$, Muscholl $M$, Holmer $S$, et al. Prevalence of left ventricular diastolic dysfunction in the community. Results from a Doppler echocar- 
diographic-based survey of a population sample. Eur Heart $J$ 2003;24:320-328.

2. Kuznetsova T, Herbots $L_{1}$ Lopez $B$, Jin $Y$, Richart T, Thijs $L_{\text {, et al. }}$ Prevalence of left ventricular diastolic dysfunction in a general population. Circ Heart Fail 2009;2:105-112.

3. Kloch-Badelek M, Kuznetsova T, Sakiewicz W, Tikhonoff V, Ryabikov A, Gonzalez $A$, et al. Prevalence of left ventricular diastolic dysfunction in European populations based on crossvalidated diagnostic thresholds. Cardiovasc Ultrasound 2012; 10:10.

4. Paulus WJ, Tschope C. A novel paradigm for heart failure with preserved ejection fraction: comorbidities drive myocardial dysfunction and remodeling through coronary microvascular endothelial inflammation. J Am Coll Cardiol 2013;62:263-271.

5. Halley CM, Houghtaling PL, Khalil MK, Thomas JD, Jaber WA. Mortality rate in patients with diastolic dysfunction and normal systolic function. Arch Intern Med 2011;171:1082-1087.

6. Kane GC, Karon BL, Mahoney DW, Redfield MM, Roger VL, Burnett JC Jr, et al. Progression of left ventricular diastolic dysfunction and risk of heart failure. JAMA 2011;306:856-863.

7. Tsang TS, Barnes ME, Gersh BJ, Takemoto Y, Rosales AG, Bailey $\mathrm{KR}$, et al. Prediction of risk for first age-related cardiovascular events in an elderly population: the incremental value of echocardiography. J Am Coll Cardiol 2003;42:1199-1205.

8. O'Rourke MF. Diastolic heart failure, diastolic left ventricular dysfunction and exercise intolerance. J Am Coll Cardiol 2001; 38:803-805.

9. Seo JY, Lee KB, Lee JG, Kim JS, Roh H, Ahn MY, et al. Implication of left ventricular diastolic dysfunction in cryptogenic ischemic stroke. Stroke 2014;45:2757-2761.

10. Kim BJ, Park JM, Kang K, Lee SJ, Kim JG, Cha JK, et al. Case characteristics, hyperacute treatment, and outcome information from the clinical research center for stroke-fifth division registry in South Korea. J Stroke 2015;17:38-53.

11. Kim BJ, Han MK, Park TH, Park SS, Lee KB, Lee BC, et al. Current status of acute stroke management in Korea: a report on a multicenter, comprehensive acute stroke registry. Int J Stroke 2014;9:514-518.

12. Lang RM, Bierig $M$, Devereux RB, Flachskampf FA, Foster $E_{\text {, }}$ Pellikka PA, et al. Recommendations for chamber quantification. Eur J Echocardiogr 2006;7:79-108.

13. Nagueh SF, Appleton CP, Gillebert TC, Marino PN, Oh JK, Smiseth $\mathrm{OA}$, et al. Recommendations for the evaluation of left ventricular diastolic function by echocardiography. Eur J Echocardiogr 2009;10:165-193.

14. Saver JL. Novel end point analytic techniques and interpreting shifts across the entire range of outcome scales in acute stroke trials. Stroke 2007;38:3055-3062.

15. Damush TM, Plue L, Bakas T, Schmid A, Williams LS. Barriers and facilitators to exercise among stroke survivors. Rehabil Nurs 2007;32:253-260, 262.

16. van Heerebeek L, Hamdani N, Falcao-Pires I, Leite-Moreira AF, Begieneman MP, Bronzwaer JG, et al. Low myocardial protein kinase $\mathrm{G}$ activity in heart failure with preserved ejection fraction. Circulation 2012;126:830-839.

17. Deswal A. Diastolic dysfunction and diastolic heart failure: mechanisms and epidemiology. Curr Cardiol Rep 2005;7:178-183.

18. Westermann D, Lindner D, Kasner M, Zietsch C, Savvatis K, Escher $F$, et al. Cardiac inflammation contributes to changes in the extracellular matrix in patients with heart failure and normal ejection fraction. Circ Heart Fail 2011;4:44-52.

19. Cumming TB, Thrift AG, Collier JM, Churilov L, Dewey HM, Donnan $G A$, et al. Very early mobilization after stroke fasttracks return to walking: further results from the phase ii avert randomized controlled trial. Stroke 2011;42:153-158.

20. Little WC, Kitzman DW, Cheng CP. Diastolic dysfunction as a cause of exercise intolerance. Heart Fail Rev 2000;5:301-306.

21. Gallanagh S, Quinn TJ, Alexander J, Walters MR. Physical activity in the prevention and treatment of stroke. ISRN Neurol $2011 ; 2011: 953818$.

22. Shih JY, Tsai WC, Huang YY, Liu YW, Lin CC, Huang YS, et al. Association of decreased left atrial strain and strain rate with stroke in chronic atrial fibrillation. J Am Soc Echocardiogr 2011; 24:513-519.

23. AlJaroudi WA, Thomas JD, Rodriguez LL, Jaber WA. Prognostic value of diastolic dysfunction: state of the art review. Cardiol Rev 2014;22:79-90.

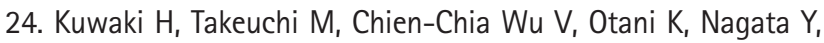
Hayashi $A$, et al. Redefining diastolic dysfunction grading: Combination of E/A $\leq 0.75$ and deceleration time $>140 \mathrm{~ms}$ and $\mathrm{E} / \varepsilon^{\prime} \geq 10$. JACC Cardiovasc Imaging 2014;7:749-758.

25. Sohn DW, Chai IH, Lee DJ, Kim HC, Kim HS, Oh BH, et al. Assessment of mitral annulus velocity by Doppler tissue imaging in the evaluation of left ventricular diastolic function. J Am Coll Cardiol 1997;30:474-480.

26. Komajda $M$, Lam CS. Heart failure with preserved ejection fraction: a clinical dilemma. Eur Heart J 2014;35:1022-1032.

27. Borlaug BA, Nishimura RA, Sorajja P, Lam CS, Redfield MM. Exercise hemodynamics enhance diagnosis of early heart failure with preserved ejection fraction. Circ Heart Fail 2010;3: 588-595. 